It may give additional orders (as indicated above) at this time. It will then direct the printer to print the desired headings. There may then follow the first CCS section. Whenever the problem tape comes to the beginning of a CCS, it delegates the control of the problem to the first routine tape. This tape now begins to move, and the machine reads from it the orders pertaining to the computation. In the course of the computation it may draw numbers from the CCS of the problem tape (in the order in which they appear there) and from the table tapes (in any desired order). The control may pass to other routine tapes. Finally it will come to an order that restores the supreme control of the problem tape. When this order is received, the routine tapes cease to move, the machine checks to see that it has received from the problem tape an indication that the present CCS section is finished, and then moves the problem tape to the beginning of the next section. If this is again a CCS, control is at once delegated to the routine tapes; if it is a SWR or SWP section, control rests with the problem tape for the length of the section. This process continues until the machine arrives at the end of the problem tape.

$$
\text { (To be concluded) }
$$

Franz L. Alt

Ballistic Research Laboratories

Aberdeen Proving Ground, Maryland

${ }^{1} M T A C$, v. 2, p. $185 f$.

2 $M T A C$, v. 2, p. $97 \mathrm{f}$, and 366.

\title{
The Square Root Method for Solving Simultaneous Linear Equations
}

The square root method for solving a system of linear equations was probably first developed by BANACHIEWICZ ${ }^{1}$ in 1938 , but it was independently developed by DwYER. ${ }^{2}$ The growing popularity of the method in the U.S. stems from the papers by Dwyer and the simple explanation of the method given by DunCAN \& KENNEY. ${ }^{3}$ The method is directly applicable to solving a system of linear equations only when the matrix of the coefficients of the unknowns is symmetric. However, if this is not the case, the system represented symbolically by the matrix equation $M X=N$, where $M$ is the matrix of the coefficients of the unknowns, $X$ is the column matrix of the unknowns, and $N$ is the column matrix of the right hand members of the equations, can be transformed into another matrix equation with a symmetric matrix as the coefficient of $X$. This is accomplished by premultiplying both sides of the equation by the inverse of $M$. The additional computations required to symmetrize the matrix $M$, before applying the square root method, makes it doubtful that this method is as efficient as other more direct methods when the coefficients of the unknowns in the original equations do not form a symmetric matrix.

The NBSCL has applied the square root method for solving systems of normal equations arising in least square solutions and has found the method very efficient. With a standard calculating machine a good computer can solve ten equations in ten unknowns carrying about eight significant digits 
(actually a fixed number of decimal places is carried) in less than three hours. The method is equally efficient for solving any system of linear equations with symmetric coefficients in spite of the fact that some of the computations may involve imaginary quantities, as will be seen later.

For a brief description of the method, consider the system

$$
\begin{aligned}
& a_{11} x_{1}+a_{12} x_{2}+\cdots+a_{1 n} x_{n}=g_{1} \\
& a_{21} x_{1}+a_{22} x_{2}+\cdots+a_{2 n} x_{n}=g_{2} \\
& \text { • } \\
& a_{n 1} x_{1}+a_{n 2} x_{2}+\cdots+a_{n n} x_{n}=g_{n}
\end{aligned}
$$

where the matrix of the coefficients, $A$, is assumed to be symmetric. This system can be represented by the matrix equation

$$
A X=G
$$

where $X$ and $G$ are the column matrices $\left[x_{i}\right]$ and $\left[g_{i}\right]$ respectively. The matrix equation (1) can be transformed into an easily solvable triangular matrix equation

$$
S X=K
$$

by defining the triangular matrix $S$ by

$$
S^{\prime} S=A
$$

where $S$ is of the form

$$
S=\left(\begin{array}{llll}
s_{11} s_{12} & \cdots & s_{1 n} \\
0 & s_{22} & \cdots & s_{2 n} \\
\cdot & & & \cdot \\
\cdot & & \cdot \\
\cdot & & & \cdot \\
0 & \cdots & 0, & s_{n n}
\end{array}\right)
$$

and $S^{\prime}$ is the inverse of $S$.

Substituting $S^{\prime} S$ for $A$ in (1), we get

$$
S^{\prime} S X=G \text {. }
$$

Therefore if $K$ is a column matrix, such that

$$
S^{\prime} K=G
$$

it follows that $S X=K$.

Once the $S$ and $K$ matrices are known, the $X$ matrix can be found very easily from the last equation.

Thus there are three steps required in the square root method. 
Step $I$-To obtain $S$ from $S^{\prime} S=A$

By equating elements of the product $S^{\prime} S$ with corresponding elements of $A$, we get $\sum_{r=1}^{i} s_{r i} s_{r j}=a_{i j}(j \geqslant i)$; therefore $s_{11}=\sqrt{a_{11}}, s_{1 j}=a_{1 j} / s_{11}$, and $s_{i i}=\left(a_{i i}-\sum_{r=1}^{i-1} s_{r i}^{2}\right)^{\frac{1}{2}}(i>1), s_{i j}=\left(a_{i j}-\sum_{r=1}^{i-1} s_{r i} s_{r j}\right) / s_{i i}(j>i)$.

Step $I I-T o$ obtain $K$ from $S^{\prime} K=G$

By equating corresponding elements of $S^{\prime} K$ and $G$, we get $\sum_{r=1}^{i} s_{r i} k_{r}=g_{i}$; therefore $k_{1}=g_{1} / s_{11}$, and $k_{i}=\left(g_{i}-\sum_{r=1}^{i-1} s_{r i} k_{r}\right) / s_{i i}(i>1)$.

Step III-To obtain $X$ from $S X=K$

By equating corresponding elements of $S X$ and $K$, we get $\sum_{r=i}^{\infty} s_{i n} x_{r}=k_{i}$; therefore $x_{n}=k_{n} / s_{n n}$, and $x_{i}=\left(k_{i}-\sum_{r=i+1}^{n} s_{i n} x_{r}\right) / s_{i i}(i<n)$.

To afford a check on the numerical work, a second system of $n$ linear equations obtained by replacing $x_{i}$ by $\bar{x}_{i}-1$ is also solved. This second matrix equation can be written as

$$
A \bar{X}=\bar{G}
$$

where the elements of $\bar{G}=\left[\overline{g_{i}}\right]$ are given by $\bar{g}_{i}=\sum_{r=1}^{n} a_{i r}+g_{i}$, which is the sum of all the coefficients plus the constant term in the $i$ th row. Since $A$ is the same as in the original matrix equation, only $\bar{K}$ and $\bar{X}$ need be computed to solve this second system. $\bar{K}$ is obtained from $S^{\prime} \bar{K}=\bar{G}$ in the same way as $K$ is obtained in Step II above, the only difference being that $g_{i}$ is replaced by $\overline{g_{i}}$ and $k_{r}$ by $\bar{k}_{r}$. $\bar{X}$ is then obtained from $S \bar{X}=\bar{K}$ as shown in Step III except that $x_{r}$ is replaced by $\overline{x_{r}}$ and $k_{i}$ by $\bar{k}_{i}$.

The computations are checked by means of the following equalities:

$$
\begin{aligned}
& \overline{k_{i}}=k_{i}+\sum_{r=i}^{n} s_{i r} \\
& \overline{x_{i}}=x_{i}+1
\end{aligned}
$$

which should be satisfied except for rounding errors.

For the checking procedure to be efficient, the computations should proceed in the following order:

(a) Obtain all the elements of $\bar{G}$.

(b) Obtain the entire first row of $S, K$ and $\bar{K}$, and check $\overline{k_{1}}$ by (5), then obtain the entire second row and check $\overline{k_{2}}$ by (5) etc., through the $n$th row.

(c) Obtain $x_{n}$ and $\overline{x_{n}}$ and check by (6), then obtain $x_{n-1}$ and $\overline{x_{n-1}}$ and check by (6), and so on until $x_{1}$ and $\overline{x_{1}}$ are obtained and checked.

In this manner, each row is checked before proceeding to the next row, and each $x$ is checked before proceeding to the next $x$.

In applying the square root method, all computations should be performed on a calculating machine writing down only the final results in the following compact and systematic arrangement: 


\begin{tabular}{|c|c|c|c|c|}
\hline$a_{11}$ & $\begin{array}{l}a_{12} \\
a_{22}\end{array}$ & $\begin{array}{ccc}a_{13} & \cdots & a_{1 n} \\
a_{23} & \cdots & a_{2 n} \\
a_{38} & \cdots & a_{3 n} \\
& & \cdot \\
& & \cdot \\
& & a_{n n}\end{array}$ & $\begin{array}{l}g_{1} \\
g_{2} \\
g_{3} \\
\dot{.} \\
\dot{.} \\
g_{n}\end{array}$ & $\begin{array}{l}\overline{\bar{g}} \\
\overline{g_{2}} \\
\overline{g_{3}} \\
\cdot \\
\dot{\cdot} \\
\overline{g_{n}}\end{array}$ \\
\hline$s_{11}$ & $\begin{array}{l}s_{12} \\
s_{22}\end{array}$ & 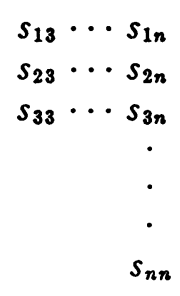 & $\begin{array}{c}k_{1} \\
k_{2} \\
k_{3} \\
\cdot \\
\dot{\cdot} \\
\dot{\cdot} \\
k_{n}\end{array}$ & $\begin{array}{c}\overline{k_{1}} \\
\frac{\overline{k_{2}}}{\overline{k_{3}}} \\
\dot{\cdot} \\
\dot{\cdot} \\
k_{n}\end{array}$ \\
\hline$\frac{x_{1}}{x_{1}}$ & $\frac{x_{2}}{x_{2}}$ & $\frac{x_{3} \cdots x_{n}}{\bar{x}_{3}} \cdots \overline{x_{n}}$ & & \\
\hline
\end{tabular}

It should be noted that the diagonal elements of the $S$ matrix involve square roots which may lead to imaginary elements. This would never occur with normal equations but could arise when $A$ is an arbitrary symmetric matrix. However, the occurrence of pure imaginary elements in $S$ causes no difficulty in applying the method.

JACK LADERMAN

NBSCL

1 Tadeusz Banachiewicz, (a) "Principes d'une nouvelle technique de la méthode des moindres carrés"; (b) "Méthode de résolution numérique des équations linéaires, du calcul des déterminants et des inverses, et de réduction des formes quadratiques," Akademija Umiejetnosci, Krakow, Wydzial Matematyczno-przyrodniczy, Bull. Intern., s.A., Sci. Math., 1938 , p. $134-135 ; 393-404$.

${ }^{2}$ P. S. DWYER, "A matrix presentation of least squares and correlation theory with matrix justification of improved methods of solution," Annals Math. Stat., v. 15, 1944, p. 82-89; and "The square root method and its use in correlation and regression," Amer. Stat. Assn., Jn., v. 40, 1945, p. 493-503.

${ }^{3}$ David B. Duncan and John F. Kenney, On the Solution of Normal Equations and Related Topics. Ann Arbor, Edwards Bros., 1946, 35 p.

\section{Coefficients for Expressing the First Twenty- Four Powers in Terms of the Legendre Polynomials}

The following table of coefficients gives the exact expression for $x^{n}$, $n=0(1) 24$, in terms of Legendre polynomials $P_{m}(x)$ (written simply as $P_{m}$ ). This extends the previous short tables (inadequate for many needs) which are given in T. M. MAcRoBert, Spherical Harmonics, London, 1927, p. 96, $n=0(1) 10$; in W. E. BYERLY, Elementary Treatise on Fourier's Series and Spherical, Cylindrical and Ellipsoidal Harmonics. Boston, 1895, p. 179, $n=0(1) 8$; and in E. W. HoBson, Theory of Spherical and Ellipsoidal 